\title{
Endosonography-guided drainage of loculated malignant ascites using double-pigtail plastic stents
}

\section{(ㄷ)(1) $\odot$}

Authors

Reiner Köck ${ }^{1}$, Christian Jürgensen ${ }^{2}$, Rupert Fischer-Lampsatis ${ }^{1}$

Institutions

1 Krankenhaus Hedwigshöhe Berlin, Medical Department, Division of Gastroenterology, Hematology and Oncology, Berlin, Germany

2 Charité Universitätsmedizin Berlin, Campus Mitte, Medical Department, Division of Hepatology and Gastroenterology, Berlin, Germany

submitted 18.2.2018

accepted after revision 3.4 .2018

\section{Bibliography}

DOI https://doi.org/10.1055/a-0605-3587 |

Endoscopy International Open 2018; 06: E902-E906

(c) Georg Thieme Verlag KG Stuttgart · New York ISSN 2364-3722

Corresponding author

Dr. med. Reiner Köck, Krankenhaus Hedwigshöhe, Medical Department, Division of Gastroenterology, Hematology and Oncology, Höhensteig 1, 12526 Berlin, Germany

Fax: +49-30-6741-5039

r.koeck@alexianer.de

\section{ABSTRACT}

Background and study aims Endoscopic ultrasound (EUS)-guided drainage is a well-established procedure for peripancreatic fluid collections (PFC) that develop in the context of acute pancreatitis or following pancreatic surgery. Malignant ascites can occur in a loculated form resembling PFC causing a variety of symptoms, with limited treatment options. There is a paucity of data about application of EUS-guided drainage for these kinds of fluid collections. So far, only one report exists describing clinical success in three cases, using fully-covered self-expanding metal stents (FCSEMS). FCSEMS, however, have possible drawbacks, particularly if they have to remain in place for an indeterminate period of time. Herein, we describe EUS-guided drainage of loculated malignant ascites using doublepigtail plastic stents in two patients severely symptomatic from esophagogastric obstruction symptoms. Technical and clinical success was achieved in both cases without clinically significant complications including one case, where ongoing symptom control could be observed after 6 months of follow-up.

\section{Introduction}

Esophageal obstruction or gastric compression caused by loculated malignant ascites are occasional clinical problems that lack a standard therapeutic approach. Development of malignant ascites usually portends a poor prognosis, with an average 6 -month survival rate of $11 \%$, except in cases of ovarian cancer, where 5 -year survival can exceed $27 \%$ [1]. If ascites occurs in a diffuse form, repeat paracentesis or tunneled catheter systems can readily be applied for palliation of symptoms. In cases of loculated ascites, however, these approaches might not be technically feasible.

Endoscopic ultrasound (EUS)-guided drainage is now a wellestablished procedure for pancreatic fluid collections (PFC) using either plastic pigtail stents or fully-covered self-expanding metal stents (FCSEMS) [2]. PFC usually occur following acute pancreatitis or pancreatic surgery. In the setting of malignant ascites, however, there are only case reports of successful application of EUS-guided drainage. Using FCSEMS, sympto- matic relief in three cases of loculated malignant ascites could be achieved [3].

We report two cases where plastic pigtails stents were being used to treat loculated malignant ascites causing esophagogastric obstruction.

\section{Case reports}

\section{Patient 1}

A 36-year-old female with stage IV rectal cancer was admitted due to nausea, dysphagia, anorexia and dyspnea. She had been diagnosed with synchronous metastatic rectal cancer 20 months earlier, and had undergone neoadjuvant chemoradiotherapy, abdominoperineal resection, partial liver resection and several lines of systemic chemotherapy. Two weeks prior to admission, she had received a radiologically placed, tunneled peritoneal drainage catheter for palliation of malignant chylous ascites. 

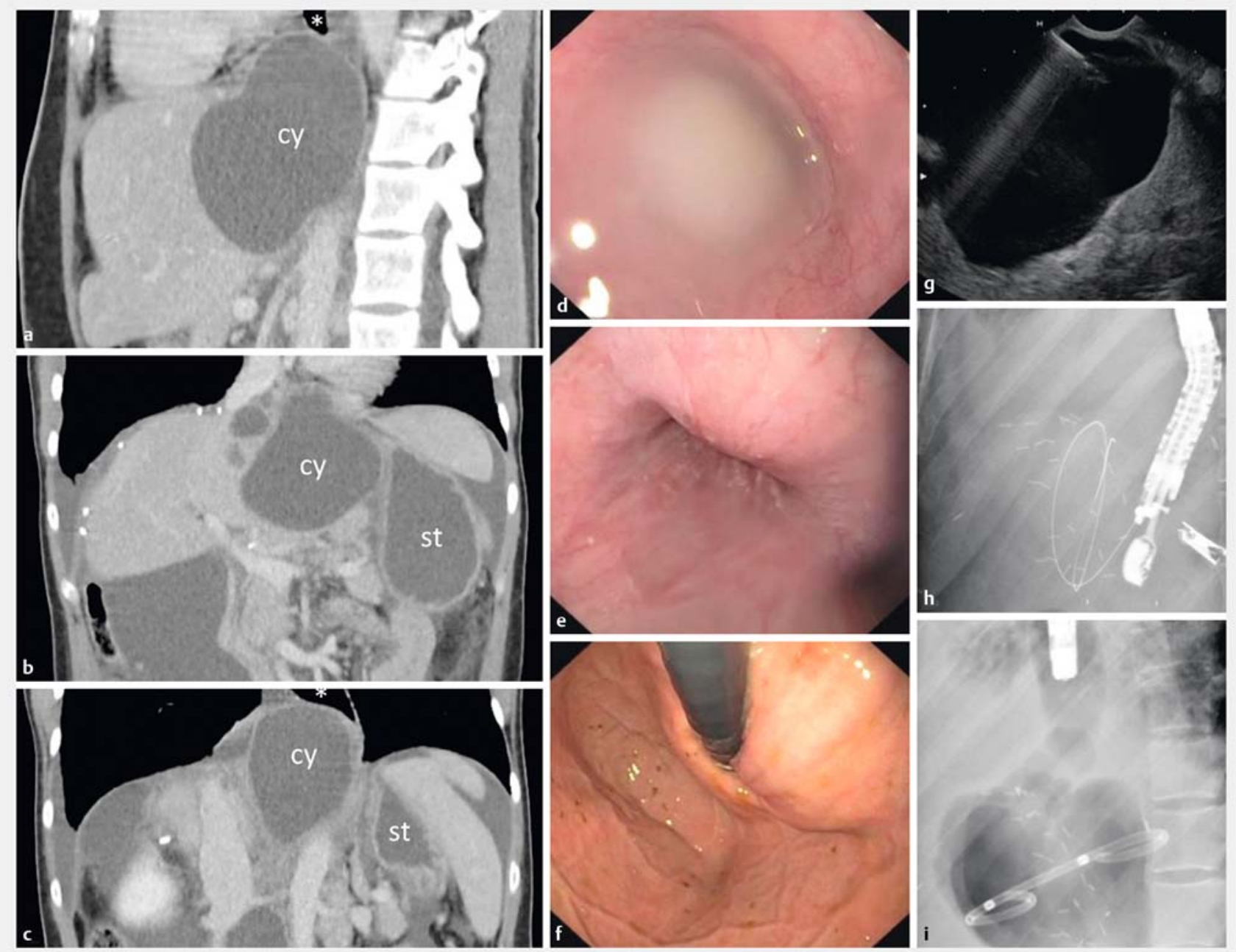

- Fig. 1 Patient 1, EUS-guided drainage of loculated ascites herniating from resected liver area to the mediastinum. a Contrast-enhanced CT scan prior to the procedure, sagittal, and $\mathbf{b}, \mathbf{c}$ coronal views, showing cyst-shaped ascites obstructing the air-filled esophagus (asterisk) with stomach (st) adjacent to the cyst (cy). Endoscopic views prior to the procedure, $\mathbf{d}$ showing trapped fluid in the esophagus oral to the stenosis and $\mathbf{e}$ the stenosis itself in the forward and $\mathbf{f}$ in the retroflexed view. $\mathbf{g}$ EUS view of puncture with a $10 \mathrm{~F}$ cystotome. Fluoroscopic views of the procedure, showing $\mathbf{h}$ linear echoendoscope with guidewire and $\mathbf{i}$ deployed double-pigtail plastic stent. Multiple staples from partial liver resection can also be seen.

Upon admission, this catheter had to be removed for infection with Staphylococcus aureus. A computed tomography (CT) scan revealed free intraperitoneal fluid next to several loculated fluid collections, one of them herniating from the epigastrium to the lower posterior mediastinum, causing compression of the esophagus at the level of the diaphragmatic hiatus. The fluid collection was inaccessible to transabdominal needle puncture due to its subdiaphragmatic position in the area of atypically resected liver segments 3 and 4 ( $\triangleright$ Fig. 1 ). A gastroscopy was performed showing total collapse of the distal esophagus caused by bulging of its left anterior wall. The stenosis could be passed with the endoscope by applying slight pressure.

Using a therapeutic echoendoscope (Pentax EG-3630U, Tokyo, Japan), an $80 \times 80-\mathrm{mm}$ almost echo-free cyst was identified as the cause of obstruction and punctured from a position approximately $2 \mathrm{~cm}$ oral to the esophagogastric junction, using a 19 G Echotip needle (Cook Medical, Limerick, Ireland). The $550 \mathrm{~mL}$ of fluid removed was slightly turbid and milky appearing. Following the procedure, the patient felt immediate relief of her dysphagia, but the symptoms had returned by the next morning. Therefore, the procedure was repeated twice with the same result each time. After discussing the experimental character of the procedure with the patient, a standard 0.035 inch guidewire was inserted into the cavity after re-puncture from the gastric cardia under fluoroscopic guidance. The cystogastrostomy tract was established using a 10 French cystotome (Cook Medical), and a 10 French $5-\mathrm{cm}$ double-pigtail plastic stent (Cook Medical) was placed into the cyst. After the procedure the patient experienced ongoing clinical improvement of her dysphagia. An episode of fever quickly resolved with anti- 

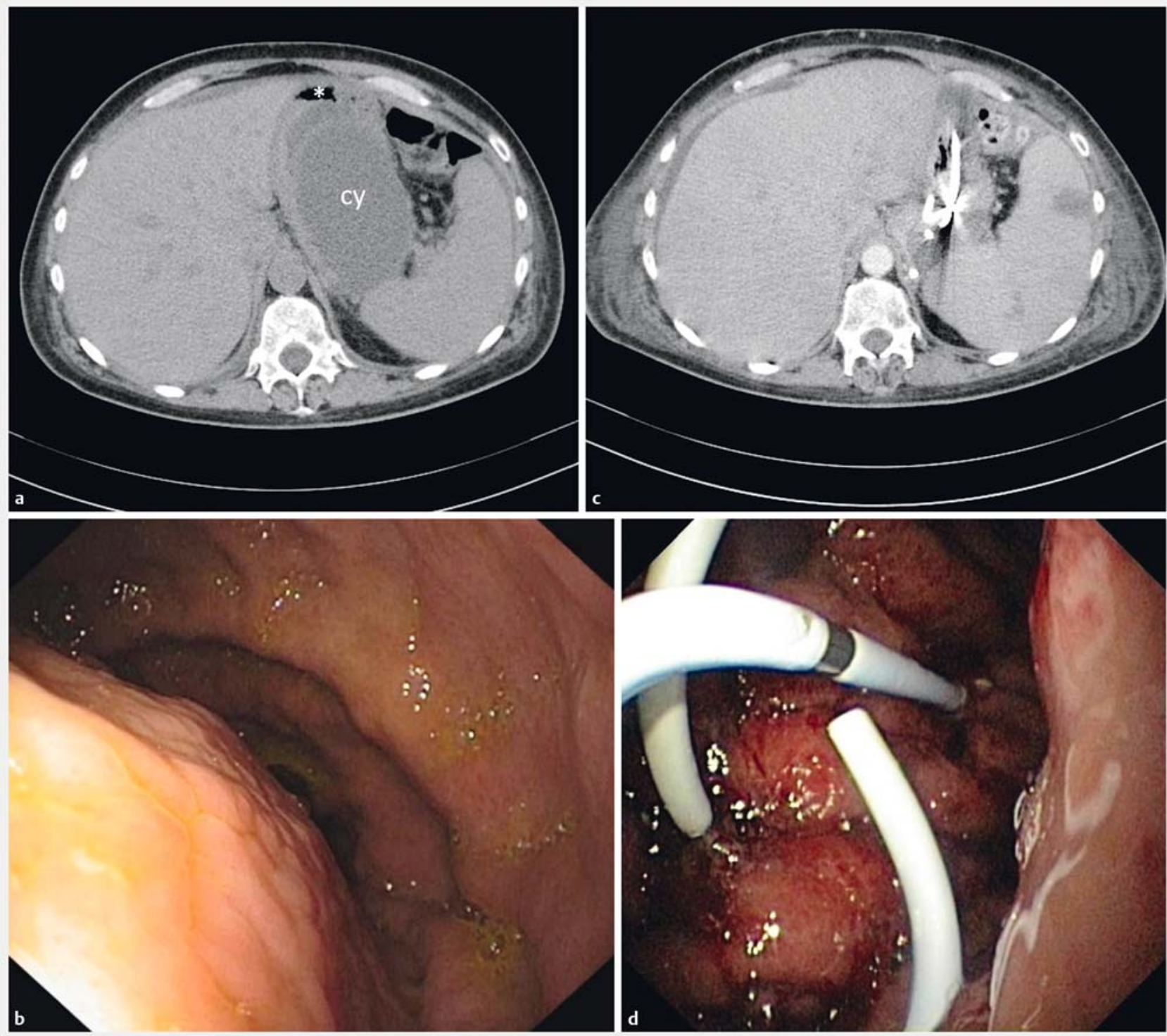

- Fig. 2 Patient 2, EUS-guided drainage of loculated ascites in the omental bursa causing near-complete constriction of the gastric cavity. a Non-enhanced CT scan prior to the procedure, showing cystic fluid collection (cy) compressing the stomach (asterisk). b Endoscopic forward view of the stomach prior to the procedure. c Contrast-enhanced CT scan (arterial phase) 2 weeks after completion of drainage, showing collapsed cyst containing plastic stents. $\mathbf{d}$ Endoscopic view immediately after placement of 2 nd stent.

biotic therapy. No other adverse events (AEs) occurred, and the pigtail drainage was left in place.

The patient died 4 weeks later from progression of her rectal cancer.

\section{Patient 2}

A 56-year-old female with progressive stage IV ovarian cancer was admitted due to nausea and vomiting to the point where even intake of oral medication was impossible. She had been diagnosed with localized cancer 7 years earlier, but had subsequently developed hepatic, lymphatic, and peritoneal spread with ascites, and received several lines of systemic chemotherapy.
Gastroscopy revealed a near-complete constriction of the gastric cavity at the level of corpus and fundus by bulging of the inferior gastric wall. This corresponded to loculated ascitic fluid in the omental bursa seen on abdominal ultrasound and CT scan ( $\$$ Fig. 2). Using a therapeutic echoendoscope (Pentax EG-3870UTK, Tokyo, Japan), the $85 \times 50-\mathrm{mm}$ echo-free cyst containing few septae could be identified and punctured as described above from a position in the gastric corpus, leaving a 10 French $5-\mathrm{cm}$ double-pigtail plastic stent in place. The procedure resulted in partial improvement of symptoms, but repeat gastroscopy still showed significant narrowing of the gastric lumen. Upon repeat EUS, only part of the loculated ascitic fluid collection appeared sufficiently drained, but another, separate 
part measuring $61 \times 34 \mathrm{~mm}$ did not. Repeat drainage with another double-pigtail stent led to radiologic complete remission of the culprit ascites pocket and enabled recommencement of oral intake. No AEs were observed.

Subsequently, another line of chemotherapy was started, leading to oncological partial remission. At the time of submission of this report, after 6 months of follow-up, the patient had progressive ascites requiring repeat paracentesis. Yet she was still in clinical remission of her gastric compression symptoms with the double-pigtail drainage left in place.

\section{Discussion}

Malignant ascites is a frequent clinical problem giving rise to a variety of symptoms such as abdominal swelling and pain, nausea, anorexia, fatigue, and dyspnea, that can significantly impair quality of life. Diuretics are of little value in this setting except for a subgroup of patients with portal hypertension due to liver metastases [4]. Some form of drainage procedure is often mandated as part of supportive care measures. Drainage methods include repeat paracentesis, permanent peritoneal ports, tunneled peritoneal catheters, central venous catheters and peritoneovenous shunts. The latter have high rates of serious AEs including mortality from pulmonary edema, disseminated intravascular coagulation and thromboembolism. Frequently used techniques are repeat paracentesis and tunneled peritoneal catheters.

We described two cases of loculated ascites that lacked apposition to the abdominal wall due to their deep epigastric position. Hence paracentesis or insertion of a peritoneal catheter did not appear safely feasible because organs like liver or stomach were interposed. Yet the patients were severely symptomatic from esophagogastric obstruction symptoms. For this particular situation of an encysted symptomatic fluid collection, we thought it appropriate to apply the method of EUSguided drainage that is well established in PFC [5]. In both scenarios, immediate contact of encapsulated fluid to the gastric or duodenal wall allows a drainage procedure that minimizes risk of spillage of intestinal contents to the free peritoneal cavity.

In the setting of malignant ascites, efficacy and safety of merely diagnostic EUS-guided paracentesis has been demonstrated [6]. EUS-guided therapeutic drainage of loculated malignant fluid collections has been reported with technical and clinical success in three cases using a lumen-apposing metal stent (LAMS) [3]. Up to now, there is no clear evidence to suggest that expandable metal stents are superior to plastic stents [5]. In an ongoing randomized trial comparing LAMS with multiple plastic stents for treatment of walled-off pancreatic necrosis, the protocol had to be changed towards early removal of LAMS due to serious AEs including bleeding [7]. The authors hypothesize that plastic stents likely gravitate towards the gastrointestinal lumen as the PFC resolves, whereas LAMS remain in place with the resultant friction against regional vasculature precipitating bleeding. In cancer patients with malignant ascites, the necessary duration of drainage is unclear at the time of stent insertion, and avoidance of complications that are related to possibly long treatment duration is of utmost importance. For these reasons, we believe that plastic pigtail stents could be more appropriate in the setting of drainage of malignant ascites. In case of viscous fluids, either LAMS or multiple plastic stents (allowing inter-stent flow) could be a therapeutic option.

Three cases of successful EUS-guided drainage using double-pigtail plastic stents to obviate the need for repeat paracentesis have recently been described in this journal [8]. In these patients malignant ascites was not described as loculated. Their follow-up was short, however, as all patients died within days to weeks after the procedure. One concern in nonloculated ascites would be that deliberately creating a connection of the gastric lumen with the free peritoneal space could promote infectious peritonitis. In our patients, however, ascites was strictly loculated thus minimizing this risk. Still, the possibility of intraperitoneal leakage is one of the drawbacks of this method, in which case we would have removed the stents. Therefore, it should be reserved for patients receiving palliative care after evaluation of alternatives. However, once the drainage is in place, the resulting peri-drainage inflammation will reduce this risk over time.

One unanswered question regards timing of stent removal. A lower incidence of recurrence of PFC without AEs like stent clogging or infection was reported in a randomized controlled trial when plastic stents were removed after 12 months compared to removal after 2 months [9]. We opted to leave stents in place as long as they appeared effective and no AEs were observed. Another concern we had prior to the procedure was whether different pressure in the thoracic and the abdominal compartment would hamper drainage of a mediastinal fluid collection through a stent inserted into the subdiaphragmatic compartment of the same collection. This, however, did not seem to prevent clinical success in the case of our patient. The chosen approach could also be applied to ascites of non-malignant origin other than PFCs. However, because there is limited experience it should be restricted to those with clear demarcation and after consideration of all other therapeutic options.

Obviously, the reported number of cases is too small to show efficacy and safety of the method. Further studies regarding EUS-guided drainage of loculated malignant ascites are needed.

\section{Conclusion}

We described for the first-time application of the well-established technique of EUS-guided drainage using plastic stents to address loculated symptomatic ascites not amenable to paracentesis. One of our patients is still in remission of her esophagogastric obstruction symptoms after 6 months of follow-up.

\section{Competing interests}

None 


\section{References}

[1] Kipps E, Tan DS, Kaye SB. Meeting the challenge of ascites in ovarian cancer: new avenues for therapy and research. Nat Rev Cancer 2013; 13: $273-282$

[2] Tyberg A, Karia K, Gabr M et al. Management of pancreatic fluid collections: A comprehensive review of the literature. World J Gastroenterol 2016; 22: 2256-2270

[3] Musumba C, Tutticci N, Nanda K et al. Endosonography-guided drainage of malignant fluid collections using lumen-apposing, fully covered self-expanding metal stents. Endoscopy 2014; 46: 690-692

[4] Stukan M. Drainage of malignant ascites: patient selection and perspectives. Cancer Manag Res 2017; 9: 115-130

[5] Fusaroli P, Jenssen C, Hocke M et al. EFSUMB Guidelines on Interventional Ultrasound (INVUS), Part V. Ultraschall Med 2016; 37: 77 - 99
[6] Montgomery MM, Leitman IM. Endoscopic ultrasound and paracentesis in the evaluation of small volume ascites in patients with intraabdominal malignancies. World J Gastroenterol 2014; 20: 10219 10222

[7] Bang JY, Hasan M, Navaneethan U et al. Lumen-apposing metal stents (LAMS) for pancreatic fluid collection (PFC) drainage: may not be business as usual. Gut 2017; 66: 2054 - 2056

[8] Romero-Castro R, Jimenez-Garcia VA, Boceta-Osuna J et al. Endoscopic ultrasound-guided placement of plastic pigtail stents for the drainage of refractory malignant ascites. Endosc Int Open 2017; 5: E1096-E1099

[9] Arvanitakis M, Delhaye M, Bali MA et al. Pancreatic-fluid collections: a randomized controlled trial regarding stent removal after endoscopic transmural drainage. Gastrointest Endosc 2007; 65: 609-619 